\title{
Valproic acid cooperates with hydralazine to augment the susceptibility of human osteosarcoma cells to Fas- and NK cell-mediated cell death
}

\author{
KOJI YAMANEGI $^{1 *}$, JUNKO YAMANE ${ }^{1 *}$, KENTA KOBAYASHI $^{1}$, NAHOKO KATO-KOGOE $^{1}$, \\ HIDEKI OHYAMA $^{1}$, KEIJI NAKASHO ${ }^{1}$, NAOKO YAMADA ${ }^{1}$, MASAKI HATA ${ }^{1}$, SATORU FUKUNAGA ${ }^{2}$, \\ HIROYUKI FUTANI $^{2}$, HARUKI OKAMURA ${ }^{3}$ and NOBUYUKI TERADA ${ }^{1}$ \\ Departments of ${ }^{1}$ Pathology, ${ }^{2}$ Orthopedic Surgery and ${ }^{3}$ Tumor Immunology and Cell Therapy, \\ Hyogo College of Medicine, Nishinomiya, Hyogo 663-8501, Japan
}

Received January 12, 2012; Accepted March 16, 2012

DOI: $10.3892 /$ ijo.2012.1438

\begin{abstract}
We investigated the effects of valproic acid (VPA), a histone deacetylase inhibitor, in combination with hydralazine, a DNA methylation inhibitor, on the expression of cell-surface Fas and MHC-class I-related chain molecules A and B (MICA and $\mathrm{B}$ ), the ligands of NKG2D which is an activating receptor of NK cells, and on production of their soluble forms in HOS, U-2 OS and SaOS-2 human osteosarcoma cell lines. We also examined the susceptibility of these cells to Fas- and NK cellmediated cell death. VPA did not increase the expression of Fas on the surface of osteosarcoma cells, while hydralazine did, and the combination of VPA with hydralazine increased the expression of cell-surface Fas. In contrast, the combination of VPA with hydralazine did not increase the production of soluble Fas by osteosarcoma cells. Both VPA and hydralazine increased the expression of cell-surface MICA and B in osteosarcoma cells, and their combination induced a greater increase in their expression. VPA inhibited the production of both soluble MICA and MICB by osteosarcoma cells while hydralazine produced no effect. Both VPA and hydralazine enhanced the susceptibility of osteosarcoma cells to Fas- and NK cell-mediated cell death and the combination of VPA with hydralazine further enhanced the effects. The present results suggest that combined administration of VPA and hydrazine is valuable for enhancing the therapeutic effects of immunotherapy for osteosarcomas.
\end{abstract}

Correspondence to: Dr Koji Yamanegi, Department of Pathology, Hyogo College of Medicine, 1-1 Mukogawa-cho, Nishinomiya, Hyogo 663-8501, Japan

E-mail: yamanegi@hyo-med.ac.jp

${ }^{*}$ Contributed equally

Key words: valproic acid, hydralazine, Fas, MHC class I-related chain molecule, osteosarcoma

\section{Introduction}

Tumor cells express MHC class I-related chain molecules A and B (MICA and B) on their cell-surface, which are ligands of the NKG2D receptor which is expressed on the cell-surface of cytotoxic immune cells, such as natural killer (NK), $\gamma \delta^{+} \mathrm{T}$ and $\mathrm{CD} 8^{+} \alpha \beta^{+} \mathrm{T}$ cells $(1,2)$. The binding of the NKG2D receptor to its ligands activates $\mathrm{NK}$ and $\gamma \delta^{+} \mathrm{T}$ cells, and co-stimulates tumor antigen-specific $\mathrm{CD}^{+} \alpha \beta^{+} \mathrm{T}$ cells $(1,2)$. Tumor cells also express the cell surface receptor Fas (CD95/APO-1), and its binding of the Fas ligand expressed on the cell-surface of NK cells or cytotoxic $\mathrm{T}$ cells induces the death of tumor cells (3-5). Thus, both the NKG2D-MICs and Fas-Fas ligand systems play important roles in the cytotoxicity of immune cells. However, tumor cells produce both soluble Fas and soluble MICs and escape from attack by cytotoxic immune cells.

The soluble Fas, which lacks the transmembrane domain of cell-surface Fas, is produced by alternative splicing of the Fas gene transcript $(6,7)$ and inhibits the binding of cell-surface Fas to the Fas ligand of cytotoxic immune cells $(6,8)$. Soluble MICs, which are produced by the proteolytic cleavage of their extracellular domain by proteases (1,9-11), interfere with the binding of MICs on the surface of tumor cells to NKG2D receptors on the surface of cytotoxic immune cells, and the binding of soluble MICs to NKG2D receptors down-regulates the NKG2D receptors on the surface of cytotoxic immune cells (1,11-14). Therefore, an increase in the expression of Fas and MICs on the surface of tumor cells, and a decrease in the production of their soluble forms by tumor cells enhance the susceptibility of tumor cells to cytotoxic immune cells.

The gene expression in tumor cells is altered by both genetic and epigenetic events, and epigenetic modifiers, such as a histone deacetylase (HDAC) inhibitor and a DNA methylation inhibitor, have been shown to suppress the growth of tumor cells and alter their gene expression profiles $(15,16)$. Several studies have demonstrated that HDAC inhibitors stimulate the expression of cell-surface MICA and MICB in a variety of tumors, enhancing the susceptibility of tumor cells to NK cell-mediated cytotoxicity (17-21). These agents also enhance the expression of cell-surface Fas $(22,23)$, and increase the 
susceptibility of tumor cells to Fas-mediated cell death (24-27). Deoxyazocytidine, a DNA methylation inhibitor, also has been reported to up-regulate the expression of Fas on colon and prostatic cancer cells, and to sensitize colon cancer cells to Fas-mediated cell death (28). Furthermore, Tang et al (29) have shown that deoxycytidine increased the cell-surface MICB expression on HEK293T and HepG2 cells and sensitized them to NK cell-mediated cytotoxicity. Thus, the use of an HDAC inhibitor alone or DNA methylation inhibitor alone has been shown to enhance the susceptibility of tumor cells to cytotoxic immune cells. However, the effects of their combination have not yet been investigated.

We have previously shown that valproic acid (VPA), a histone deacetylase inhibitor, does not increase the expression of Fas on the cell-surface of human osteosarcoma cells, but sensitizes them to Fas-mediated cell death and decreases their production of soluble Fas (27). Furthermore, we have shown that VPA increases the expression of MICA and B on the cell-surface of human osteosarcoma cells, sensitizes them to NK cell-mediated cell death and decreases their production of soluble MICA and MICB (21). In the present study, we investigated the effects of a combination of VPA and a DNA methylation inhibitor on the expression of Fas and MICA and B on the cell-surface of osteosarcoma cells, their susceptibility to Fas- and NK cell-mediated cell death, and their production of soluble Fas and soluble MICA and MICB. We adopted VPA and hydralazine as a representative histone deacetylase inhibitor and DNA methylation inhibitor, respectively $(15,30)$, because VPA and hydralazine are currently being used for the treatment of epilepsy and mood disorders $(30,31)$ and for anti-hypertensive therapy (32), respectively, without serious side effects.

\section{Materials and methods}

Reagents and antibodies. Sodium valproate was purchased from Wako (Osaka, Japan), hydralazine hydrochloride was from Sigma-Aldrich (St. Louis, MI, USA), the PE-conjugated antihuman Fas mouse monoclonal antibody $\left(\mathrm{IgG}_{1}\right)$ was from BD Pharmingen (San Jose, CA, USA), the PE-conjugated anti-human MICA and B mouse monoclonal antibody $\left(\operatorname{IgG}_{2 b}\right)$ was from $R \& D$ Systems (Minneapolis, MN, USA), the agonistic anti-human Fas mouse monoclonal antibody (IgM) (CH-11) was from MBL (Nagoya, Japan), and the purified anti-human NKG2D mouse monoclonal antibody $\left(\operatorname{IgG}_{1}\right)$ and control mouse $\operatorname{IgM}, \operatorname{IgG}_{1}$ and $\mathrm{IgG}_{2 \mathrm{~b}}$, were purchased from BioLegend (San Diego, CA, USA).

Cells. U-2 OS human osteosarcoma cells and human natural killer NK-92 cells were purchased from the American Type Culture Collection (ATCC, Manassas, VA, USA), and HOS and SaOS-2 human osteosarcoma cells were purchased from Riken BRC Cell Bank (Tsukuba, Japan). The HOS cells were cultured in Eagle's minimum essential medium (Invitrogen, Carlsbad, CA, USA), and the U-2 OS and SaOS-2 cells in McCoy's 5A modified medium (Invitrogen). All these media contained 10\% fetal bovine serum (FBS) (MP Biomedical, Morgan Irvine, CA, USA), penicillin $(100 \mathrm{U} / \mathrm{ml})$ and streptomycin $(100 \mu \mathrm{g} / \mathrm{ml})$. The NK-92 cells were cultured in minimum essential $\alpha$ medium (Invitrogen) containing $0.2 \mathrm{mM}$ inositol, $0.1 \mathrm{mM}$ 2-mercaptoethanol, $0.02 \mathrm{mM}$ folic acid, $17 \mathrm{mM}$ sodium bicarbonate, $12.5 \%$ horse serum (Invitrogen), $12.5 \%$ FBS and $100 \mathrm{U} / \mathrm{ml}$ recombinant human interleukin 2 (Peprotech, Rock Hill, NJ, USA). All cells were cultured in a humidified atmosphere of $5 \% \mathrm{CO}_{2}$ in air at $37^{\circ} \mathrm{C}$.

Cell growth. HOS and U-2 OS cells ( $1 \times 10^{3}$ cells per well), and SaOS- 2 cells $\left(2 \times 10^{3}\right.$ cells per well) were seeded in 96 welltissue culture plates containing $100 \mu \mathrm{l}$ of medium in each well. After $24 \mathrm{~h}$ (day 0), VPA $(1.0 \mathrm{mM})$, hydralazine $(10 \mu \mathrm{M})$ or a combination of both agents was added to the medium, and the cultured for another 7 days with a medium change on day 3 . The number of viable cells in each well was estimated using a CellTiter $96^{\circledR} \mathrm{AQ}_{\text {ueous }}$ One solution cell proliferation assay kit (Promega, Madison, WI, USA), according to the manufacturer's instructions.

Flow cytometric analysis. HOS, U-2 OS and SaOS-2 cells were seeded at 2, 2 and $4 \times 10^{3}$ cells/dish, respectively, in $6 \mathrm{~cm}$-tissue culture dishes containing $4 \mathrm{ml}$ of medium per dish. After $24 \mathrm{~h}$ (day 0$)$, VPA $(1.0 \mathrm{mM})$, hydralazine $(10 \mu \mathrm{M})$ or a combination of both agents was added to medium, and the cells were cultured for another 7 days, with a medium change on day 3 . After the culture, cells were detached from the dishes, and their expression of membrane-bound Fas and membrane-bound MICA and B was analyzed by a flow cytometric analysis, as described previously $(21,27)$. The percentages of membrane-bound Fasand MICA and B-positive cells were determined by the flow cytometric analysis, and the effects of VPA, hydralazine or the combination were evaluated by determining the ratio of the percentage of positive cells in treated cultures to the average percentage of positive cells in untreated cultures.

Enzyme-linked immunosorbent assays (ELISAs) . HOS, U-2 OS and SaOS-2 cells were seeded at 1,1 and $2 \times 10^{5}$ cells/dish, respectively, in $10 \mathrm{~cm}$-tissue culture dishes containing $5 \mathrm{ml}$ of medium per dish. After $24 \mathrm{~h}$ (day 0), VPA (1.0 mM), hydralazine $(10 \mu \mathrm{M})$ or a combination of both agents was added to the medium, and the cells were cultured for another 7 days, with a medium change on day 3. After being cultured, the medium was collected for an assay of the soluble Fas, soluble MICA and soluble MICB levels using ELISA systems for human soluble Fas, MICA and MICB (R\&D Systems) and a microplate reader (Bio-Rad, Tokyo, Japan). The effects of VPA, hydralazine and the combination were evaluated by determining the amount of the soluble forms of the proteins per $10^{4}$ viable cells in treated cultures as a percentage of the average value in untreated cultures.

Cell death induced by an agonistic anti-Fas antibody. HOS, U-2 OS and SaOS-2 cells were seeded at 2, 2 and $4 \times 10^{4}$ cells/ dish, respectively, in $6 \mathrm{~cm}$-tissue culture dishes containing $4 \mathrm{ml}$ of medium per dish. After $24 \mathrm{~h}$ (day 0), VPA (1.0 mM), hydralazine $(10 \mu \mathrm{M})$ or a combination of both agents was added to the medium, and cells were cultured for another 7 days, with a medium change on day 3. After the culture, the dishes in each group (control, VPA, hydralazine or VPA and hydralazine) were divided into 2 groups, the media in all dishes were removed, and the cells were washed with medium without VPA or hydralazine two times. Then, one group received medium with $100 \mathrm{ng} / \mathrm{ml}$ of an agonistic anti-human Fas mouse monoclonal antibody (IgM) (CH-11), and the other group received medium with mouse IgM 
as a control. The cells were cultured for another $16 \mathrm{~h}$, and the number of viable cells per dish was determined by the trypan blue exclusion test. The effect of the agonistic anti-Fas antibody on cell death was evaluated by expressing the number of viable cells per dish after treatment with the agonistic anti-Fas antibody as the percentage of the average number of viable cells per dish after treatment with $\operatorname{IgM}$.

Cytotoxicity assay. The NK cell cytotoxicity against the target cells (HOS, U-2 OS and SaOS-2 osteosarcoma cells) was analyzed using a CytoTox-Fluor ${ }^{\mathrm{TM}}$ Cytotoxicity Assay kit (Promega), according to the manufacturer's instructions. The osteosarcoma target cells (T) were cultured in medium with or without VPA $(1.0 \mathrm{mM})$, hydralazine $(10 \mu \mathrm{M})$ or a combination of both agents for 7 days. Then, the NK cell cytotoxicity assay was carried out by incubating these cells with NK-92 effector cells (E) at various T:E ratios $(1 / 20-1 / 5)$ for $3 \mathrm{~h}$ at $37^{\circ} \mathrm{C}$. In blocking experiments, the NK-92 cells were cultured in medium with $1.0 \mu \mathrm{g} / \mathrm{ml}$ of the purified anti-human NKG2D antibody for $16 \mathrm{~h}$ before incubating them with the target cells. The number of dead cells was estimated by measuring the activity of proteases in the medium which were released from dead cells. The protease activity in the medium that was released spontaneously from target cells, and the total maximum protease activity released from all target cells by treatment with $1 \%$ Triton X-100 was also determined. The percentage of target cells killed by NK-92 cells was calculated as follows: $100 \mathrm{x}$ (sample protease activity-spontaneously released protease activity)/(total protease activity-spontaneously released protease activity).

Methylation-specific restriction enzyme digestion PCR. SaOS-2 cells were cultured in medium with or without $1.0 \mathrm{mM}$ VPA, $10 \mu \mathrm{M}$ hydralazine or a combination of both agents for 7 days, with a medium change on day 3 . The genomic DNA of cells was extracted using a Wizard ${ }^{\circledR}$ SV genomic DNA purification system (Promega). An aliquot of each extracted DNA sample was digested by methylation-sensitive HpaII or the methylation-insensitive MspI restriction enzyme (New England Biolabs, Beverly, MA, USA). After digestion, each DNA sample was amplified by PCR using Ex Taq DNA polymerase (Takara Bio Inc., Ohtsu, Shiga, Japan) and the following primers: sense, 5'-ACGAACCCTGACTCCTTCCT-3' and antisense, 5'-AG CTTCCCCAACTCCGTACT-3' for the Fas promoter region (-386 to -30); sense, 5'-GAGCCGGTTTAATGGGGCGG-3' and antisense, 5'-CAGAAAATGGGGAGCACGCG-3' for the MICA promoter region (-420 to -184); sense, 5'-AAGGA ACAAGCCAGTGAGAG-3' and antisense, 5'-AACCCGTGG AAATTTAGTCA-3' for the MICB promoter region (-437 to -64). The initial denaturation and final extension of PCR were done at $95^{\circ} \mathrm{C}$ for $5 \mathrm{~min}$ and at $72^{\circ} \mathrm{C}$ for $5 \mathrm{~min}$, respectively, and the amplification conditions of the PCR were 35 cycles at $95^{\circ} \mathrm{C}$ for $30 \mathrm{sec}, 57^{\circ} \mathrm{C}$ for $30 \mathrm{sec}$, and $72^{\circ} \mathrm{C}$ for $45 \mathrm{sec}$ for the Fas, MICA and MICB promoter regions. The amplified fragments were resolved by electrophoresis on $2.0 \%$ agarose gels, and were detected by ethidium bromide staining.

Statistical analysis. The data are presented as the means \pm SEM. The data from 3 or more groups were analyzed by the two-tailed Dunnett's t-test for multiple comparisons. A P-value $<0.05$ was considered to be significant.

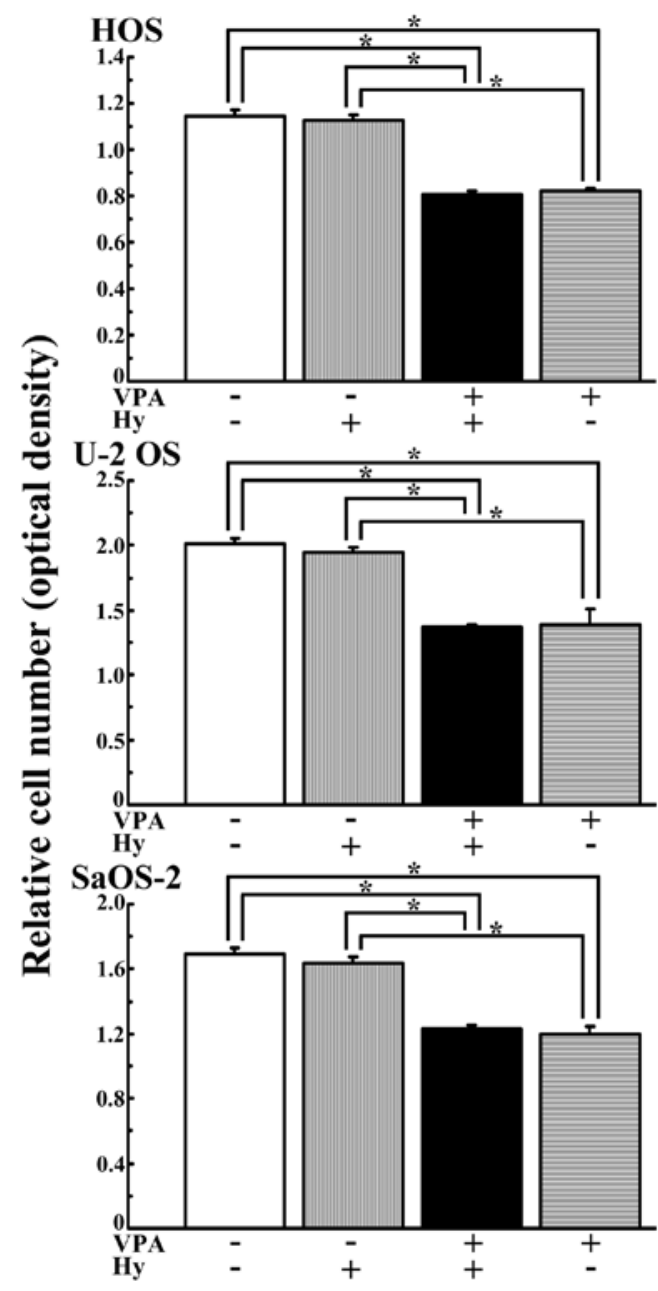

Figure 1. The effects of VPA, hydralazine and their combination on cell growth. Osteosarcoma cells were cultured in medium with or without $1.0 \mathrm{mM}$ VPA, $10 \mu \mathrm{M}$ hydralazine (Hy) or a combination of both agents for 7 days. Each bar indicates the mean \pm SEM of 16 wells. ${ }^{*} \mathrm{P}<0.05$, significant difference.

\section{Results}

Growth of osteosarcoma cells. VPA at $1.0 \mathrm{mM}$ inhibited the growth of the three osteosarcoma cell lines (HOS, U-2 OS and SaOS-2 cells) by about $25-30 \%$ on day 7 in culture. However, hydralazine at $10 \mu \mathrm{M}$ showed no significant effect on their growth, and did not decrease the cell number any further when used in combination with VPA (Fig. 1).

The expression of Fas on the surface of osteosarcoma cells. A flow cytometric analysis showed that cell-surface Fas was expressed in 17, 18 and 51\% of HOS, U-2OS and SaOS-2 cells cultured in the control medium for 7 days, respectively. Fig. 2A shows the effects of VPA $(1.0 \mathrm{mM})$, hydralazine $(10 \mu \mathrm{M})$ and their combination on the expression of cell-surface Fas after 7 days of culture. VPA showed no significant effect on the expression of cell-surface Fas in any of the three cell lines, but hydralazine increased the expression of cell-surface Fas in HOS, U-2OS and SaOS-2 cells about 2-, 2- and 1.4-fold, respectively. The combination of the agents led to further augmentation of the expression of cell-surface Fas by these cells, leading to increases 
(A)
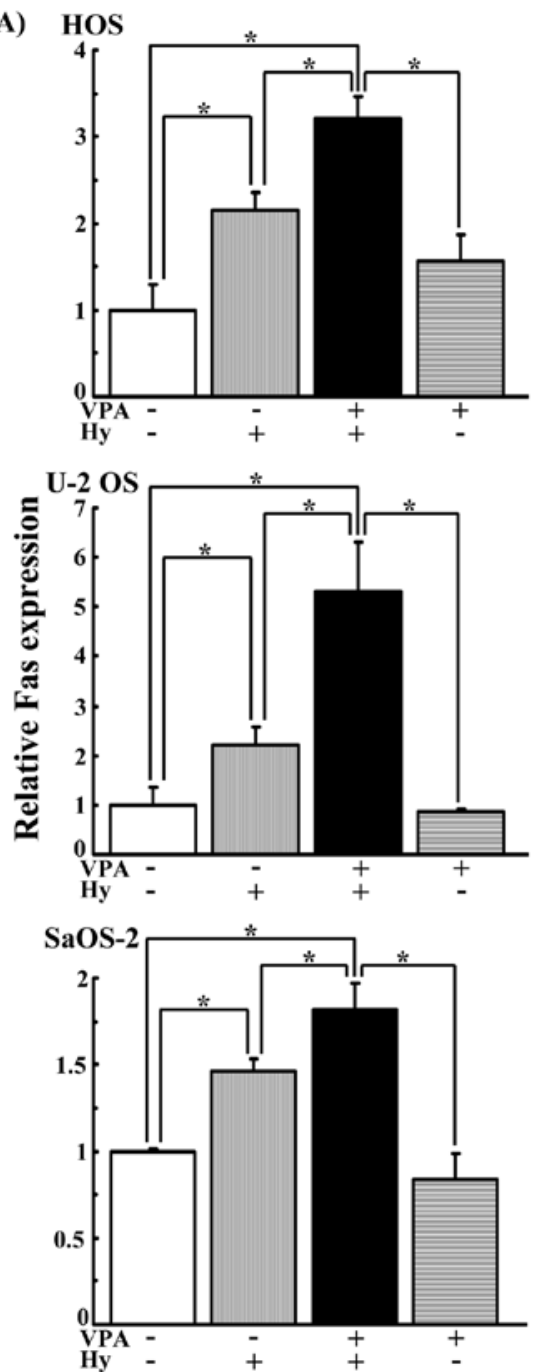

(B) HOS
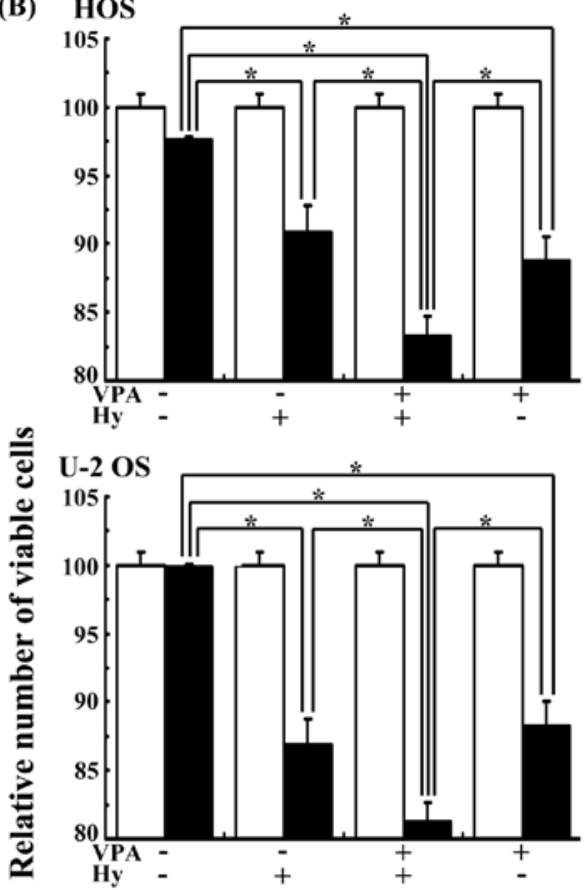

SaOS-2

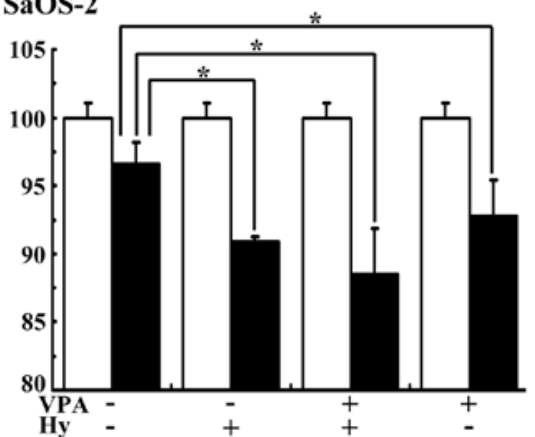

Figure 2. The effects of VPA, hydralazine and their combination on the expression of cell-surface Fas and Fas-mediated cell death. Osteosarcoma cells were cultured in medium with or without $1.0 \mathrm{mM}$ VPA, $10 \mu \mathrm{M}$ hydralazine (Hy) or a combination of both agents for 7 days. (A) The expression of cell-surface Fas was analyzed by flow cytometry, and is shown as the ratio of a percentage of positive cells in treated cultures to the average percentage of positive cells in control cultures. Each bar indicates the mean \pm SEM of 4 flow cytometric profiles. ${ }^{*} \mathrm{P}<0.05$, significant difference. (B) Fas-mediated cell death. On day 7 of culture, culture dishes were divided into 2 groups, and the medium was exchanged for fresh medium containing an agonistic anti-Fas antibody (IgM) or control IgM. After $16 \mathrm{~h}$, the number of viable cells per dish was determined. The number of viable cells is shown as the percentage of the average number of cells in culture with the control IgM. Each bar indicates the mean \pm SEM of 4 dishes. $\mathrm{P}<0.05$, significant difference.

in HOS, U-2OS and SaOS-2 cells about 3.2-, 5.3- and 1.8-fold, respectively (Fig. 2A).

Cell death induced by the agonistic anti-Fas antibody. Osteosarcoma cells were cultured under various conditions for 7 days. On day 7, the medium was replaced with fresh medium containing an agonistic anti-Fas antibody (IgM) or control $\mathrm{IgM}$, and after culture for $16 \mathrm{~h}$, the number of viable cells was determined (Fig. 2B). VPA alone and hydralazine alone both significantly enhanced the Fas-mediated cell death of the three osteosarcoma cell lines, and the combination of VPA and hydralazine further augmented the Fas-induced cell death of HOS and U-2 OS cells.

Secretion of soluble Fas by osteosarcoma cells. The osteosarcoma cells were cultured for 7 days under various conditions, and the amount ( $\mu \mathrm{g}$ per $10^{4}$ viable cells) of soluble Fas secreted in the medium during the last 4 days (from day 3 to 7) was estimated (Fig. 3). VPA significantly decreased the amount of soluble Fas secreted by HOS, U-2 OS and SaOS- 2 cells by $10-20 \%$, while hydralazine significantly increased it by $30-50 \%$. The combination of VPA and hydralazine did not significantly change the amount of soluble Fas secreted by any of the osteosarcoma cell lines.

The expression of MICA and B on the surface of osteosarcoma cells. Osteosarcoma cells were cultured under various conditions for 7 days, and the cell-surface expression of MICA and B was examined by flow cytometry (Fig. 4A). Cell-surface MICA and $\mathrm{B}$ were expressed in 5.3, 8.1 and $2.8 \%$ of HOS, U2-OS and SaOS-2 cells, respectively. VPA increased the expression of MICA and B on the cell-surface of HOS, U-2OS and SaOS-2 cells about 2.5-, 5.5- and 5.7-fold, respectively, while hydralazine increased the expression about 3.5-, 1.5- and 2.3-fold, 


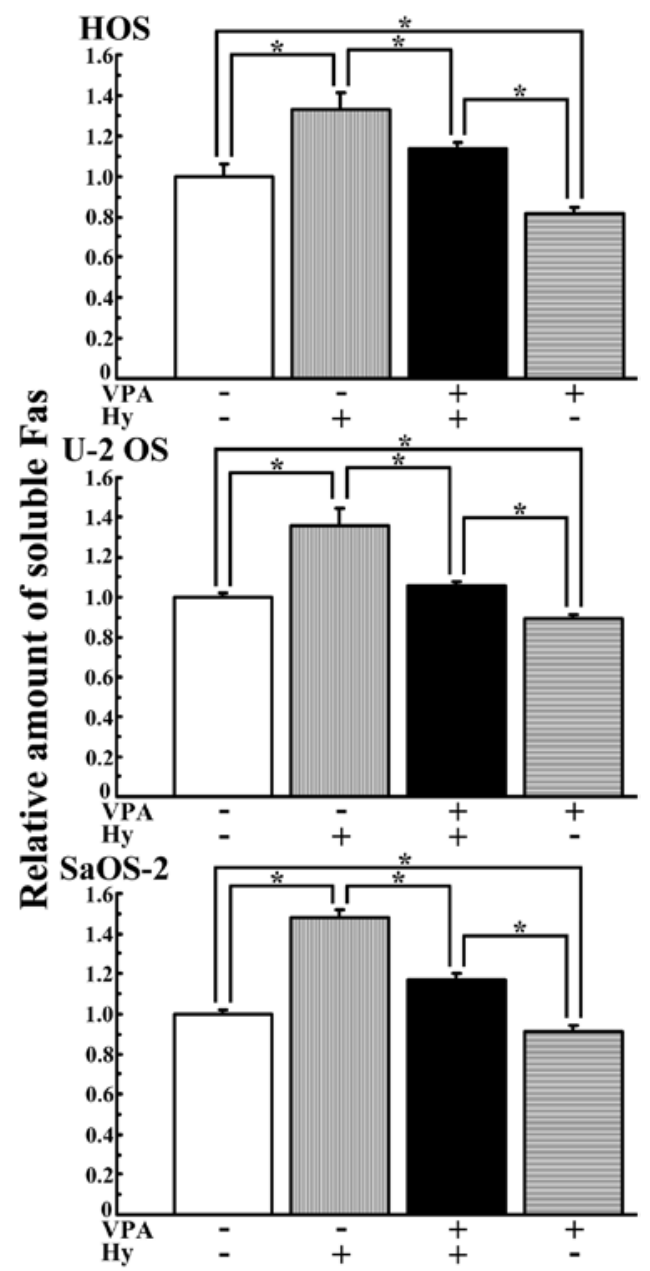

Figure 3. The effects of VPA, hydralazine and their combination on the secretion of soluble Fas. Osteosarcoma cells were cultured in medium with or without $1.0 \mathrm{mM}$ VPA, $10 \mu \mathrm{M}$ hydralazine (Hy) or a combination of both agents for 7 days, with a medium change on day 3 . The amount of soluble Fas accumulated in the medium during the last 4 days in culture was measured, and the amount of soluble Fas per $10^{4}$ viable cells was determined. The value was expressed as a ratio to the average amount of soluble Fas per $10^{4}$ viable cells in control cultures. Each bar indicates the mean \pm SEM of 8 dishes. ${ }^{*} \mathrm{P}<0.05$, significant difference.

respectively. Hydralazine further enhanced the VPA-induced expression of MICA and B on the cell-surface of HOS and SaOS-2 cells.

NK cell-mediated cytotoxicity. The three osteosarcoma cell lines were cultured under various conditions for 7 days, and their susceptibility to the cytotoxicity of NK-92 cells, an NK cell line, was examined (Fig. 4B). VPA alone or hydralazine alone increased the susceptibility of all three cell lines to the NK-92 cells. The combination of VPA and hydralazine further increased the cytotoxicity, and pretreatment of the NK-92 cells with an NKG2D-blocking antibody completely prevented the cytotoxic activity of NK-92 cells against the osteosarcoma cells treated with the combination of VPA and hydralazine.

Secretion of soluble MICA and B. The amount (per $10^{4}$ viable cells) of soluble MICA or MICB (Fig. 5) in the medium of osteosarcoma cells during the last 4 days of a 7-day culture (from day 3 to 7) was estimated. VPA significantly decreased the amounts of both soluble MICA and MICB in the culture media of all osteosarcoma cell lines, but hydralazine both alone or in the presence of VPA showed no significant effect on the amount of soluble MICA and MICB.

The effect of the methylation status of the Fas, MICA and $M I C B$ gene promoters. After 7 days of culture, the DNA was extracted from SaOS-2 cells. The DNA samples were digested with HpaII, a methylation-specific restriction enzyme, and the promoter regions of Fas, MICA and MICB were amplified by PCR (Fig. 6). Hydralazine, but not VPA, demethylated the promoter region of the Fas gene. On the other hand, the examined promoter regions of the MICA and MICB genes were not methylated, and we did not observe any effect of hydralazine on the DNA methylation of these promoter regions. VPA (1.0 $\mathrm{mM})$, but not hydralazine, exerted activity as a histone deacetylase inhibitor, as previously reported $(21,27)$ (data not shown).

\section{Discussion}

VPA inhibited the growth of osteosarcoma cells and their secretion of soluble Fas and soluble MICA and MICB, and increased the susceptibility of the osteosarcoma cells to Fas- and NK cellmediated cell death. These results confirmed the results of our previous reports $(21,27)$, which showed that VPA increased the acetylation of histones, and that Trichostatin A, another histone deacetylase inhibitor, exerted effects similar to those of VPA, suggesting that the effects of VPA can be ascribed to its actions as a histone deacetylase inhibitor.

Hydralazine increased the expression of Fas and MICA and B on the surface of osteosarcoma cells. Petak et al (28) have shown that deoxyazocytidine, another DNA methylation inhibitor, down-regulates the methylation of the p53-binding region of the Fas gene of colon cancer cells, and up-regulates the expression of Fas on their cell-surface, sensitizing them to Fas-mediated cell death. In the present study, hydralazine demethylated the promoter region of the Fas gene of osteosarcoma cells. Therefore, the effect of hydralazine on the expression of Fas seems to be due to its action as a DNA methylation inhibitor. Furthermore, Tang et al (29) have shown that deoxyazocytidine induced demethylation at the MICB promoter region of HEK293T and HepG2 cells, up-regulated MICB on their cell-surface, and sensitized the cells to NK-cell-mediated cell death. In the present study, hydralazine showed the same effects as deoxyazocytidine with regard to the expression of MICA and B and the sensitization to NK cells. However, the promoter regions of MICA and MICB, which contain many potential regulatory elements and sites (35), were not methylated in our osteosarcoma cells. Therefore, the mechanism by which hydralazine increased the expression of MICA and B is still unknown. However, it is possible that hydralazine increased the expression of MICA and B through enhancement of the expression of transcription factors as a result of its inhibition of DNA methylation.

In the present study, VPA and hydralazine cooperated to increase the expression of Fas and MICA and B on the surface of osteosarcoma cells, and these effects were greater than those 
(A)
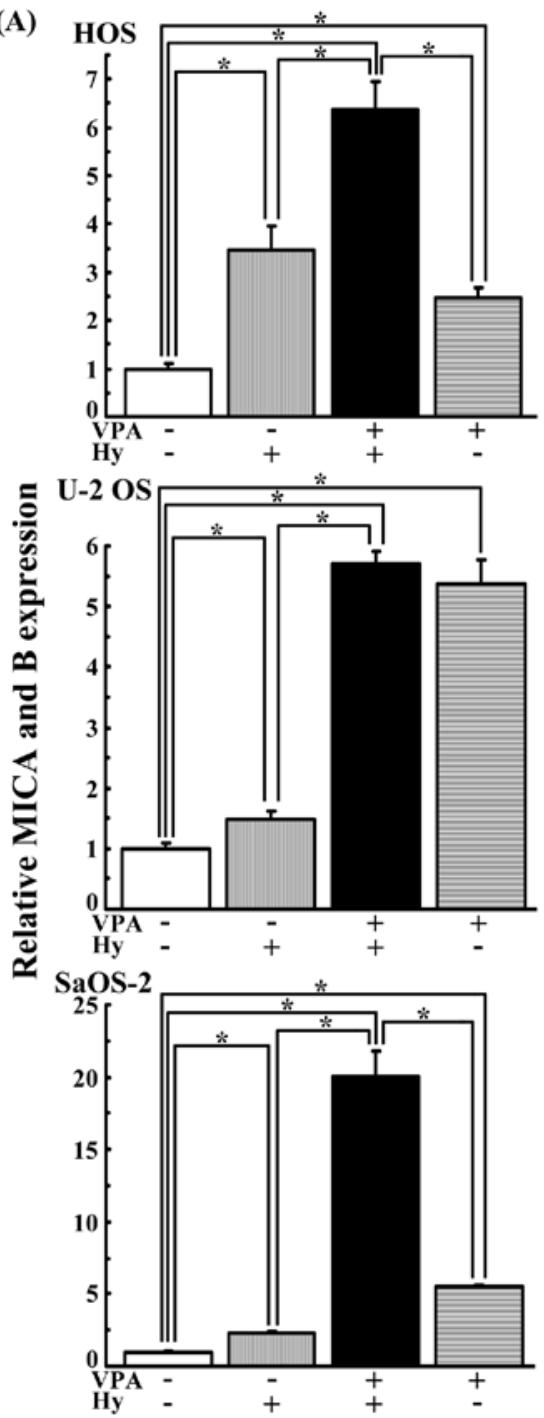

(B)

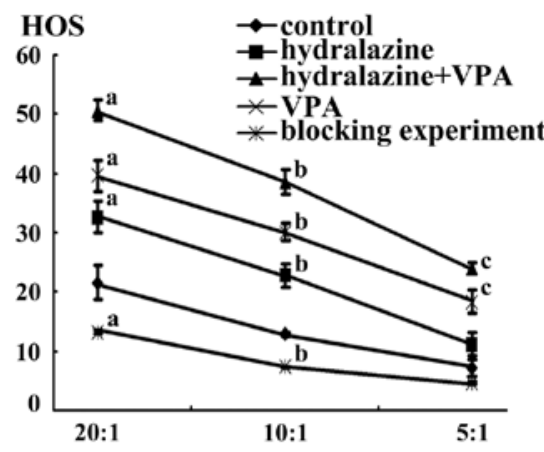

U-2 OS

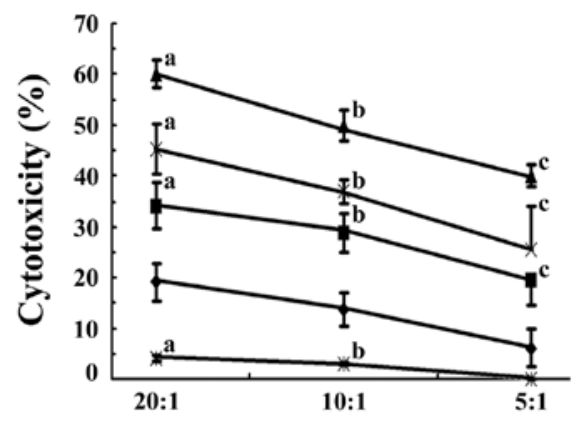

SaOS-2

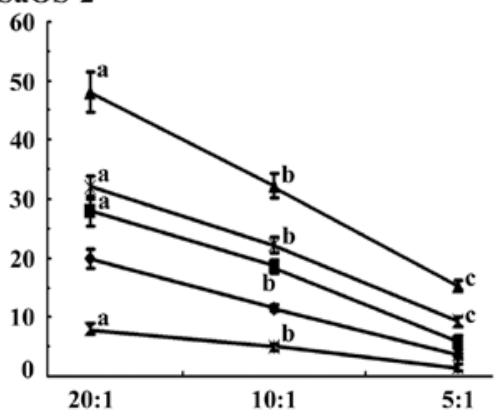

Figure 4. The effects of VPA, hydralazine and their combination on the expression of cell-surface MICA and B and NK cell-mediated cell death. Osteosarcoma cells were cultured in medium with or without $1.0 \mathrm{mM} \mathrm{VPA}, 10 \mu \mathrm{M}$ hydralazine or a combination of both agents for 7 days. (A) The expression of cell-surface MICA and B was analyzed by flow cytometry using an antibody that recognizes both MICA and MICB, and is shown as the ratio of a percentage of positive cells in treated cultures to the average percentage of positive cells in control cultures. Each bar indicates the mean \pm SEM of 4 flow cytometric profiles. $\mathrm{P}<0.05$, significant difference. (B) NK cell-mediated cell death. On day 7 of culture, osteosarcoma cells (target cells, T) and NK-92 cells (effector cells, E) were incubated for 3 h at various ratios. In blocking experiments, the NK-92 cells were incubated with an anti-NKG2D antibody for $16 \mathrm{~h}$ prior to incubation with the osteosarcoma cells cultured with VPA and hydralazine. Each point indicates the mean \pm SEM of 8 samples. ${ }^{\mathrm{a}, \mathrm{b}, \mathrm{c}} \mathrm{P}<0.05$, significant difference compared to the values of control cultures.

exerted by VPA or hydralazine alone. The cooperation of VPA and hydralazine seems to be ascribed to augmented transcription of the Fas gene caused by both the demethylation of the Fas promoter region and histone deacetylation. In addition, VPA and hydralazine cooperated to increase the susceptibility of osteosarcoma cells to NK cell-mediated cytotoxicity. Since the NK cell-mediated cytotoxic activity was abolished by pretreatment of the NK cells with an NKG2D receptor blocking antibody, this increase in activity was ascribed to the increased MICA and MICB induced by VPA and hydralazine although the mechanism of their cooperation is unknown.

VPA sensitized osteosarcoma cells to Fas-mediated cell death without increasing the expression of their cell-surface Fas. It was previously reported that FR901228, a histone deacetylase inhibitor, sensitized human osteosarcoma cells to Fas-mediated cell death $(24,25)$. Both FR901228 and VPA have been shown to down-regulate the expression of FLIP, an inhibitor of Fas-mediated activation of caspase-8 $(25,26)$, which is likely involved in the sensitization of human osteosarcoma cells to Fas-mediated cell death. Hydralazine alone also sensitized osteosarcoma cells to Fas-mediated cell death, and hydralazine in combination with VPA produced a greater effect compared to hydralazine or VPA alone. The effect of hydralazine seems to be partly due to its augmentation of the expression of cell-surface Fas, but other unknown actions of hydralazine on the Fas-mediated cell death pathway may be involved.

Hydralazine increased both the expression of cell-surface Fas and the production of soluble Fas. Since soluble Fas is produced by alternative splicing of the Fas gene transcript $(6,7)$, this effect of hydralazine seems to be associated to its augmenting effect on the expression of cell-surface Fas. On the other hand, VPA decreased the production of soluble Fas with no significant effect on the expression of the cell-surface Fas. Furthermore, 

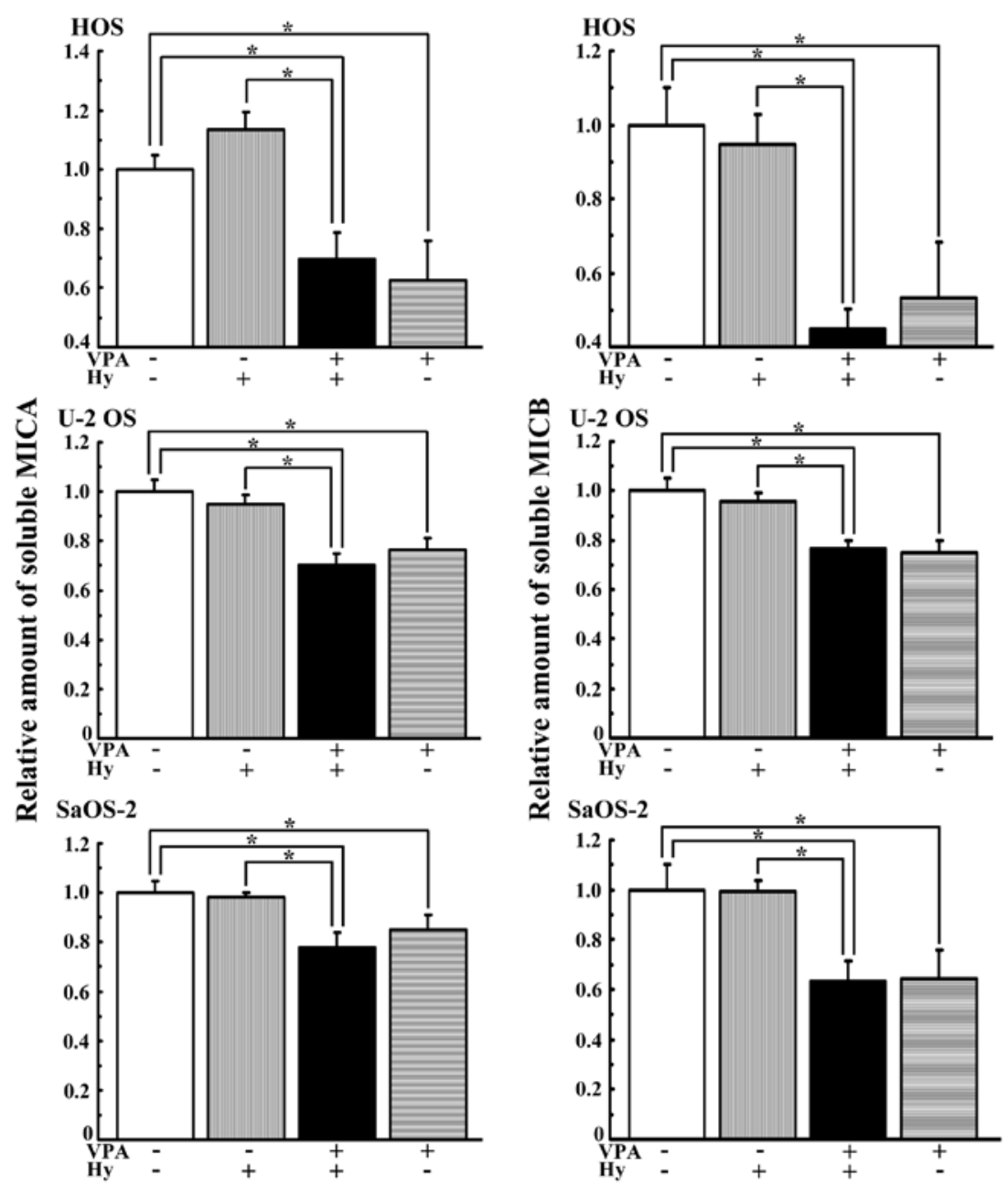

Figure 5. The effects of VPA, hydralazine and their combination on the production of soluble MICA and MICB. Osteosarcoma cells were cultured in medium with or without $1.0 \mathrm{mM}$ VPA, $10 \mu \mathrm{M}$ hydralazine or a combination of both agents for 7 days, with a medium change on day 3 . The amount of soluble MICA and MICB accumulated in the medium during the last 4 days was measured, and the amount of soluble MICA or MICB per $10^{4}$ viable cells was determined. The value was expressed as a ratio to the average amount of soluble MICA or MICB per $10^{4}$ viable cells in control cultures. Each bar indicates the mean \pm SEM of 8 dishes. ${ }^{*} \mathrm{P}<0.05$, significant difference.

\section{SaOS-2}

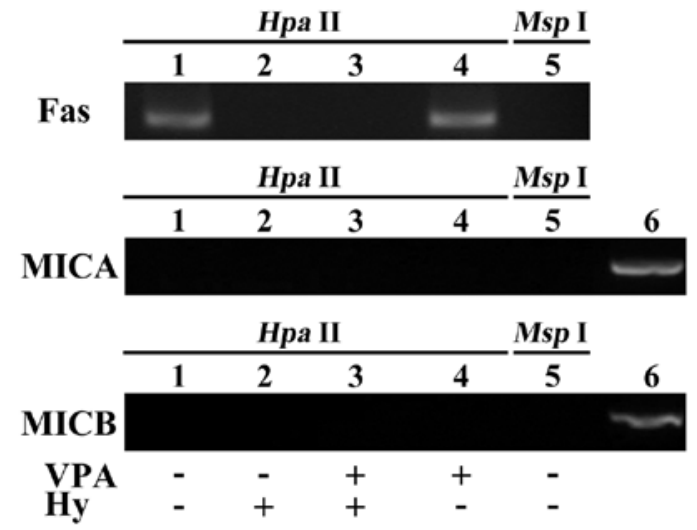

Figure 6. The effects of VPA, hydralazine and their combination on the methylation of the promoter regions of the Fas, MICA and MICB genes. SaOS-2 cells were cultured in medium with or without $1.0 \mathrm{mM} \mathrm{VPA}, 10 \mu \mathrm{M}$ hydralazine or a combination of both agents for 7 days. DNA from cells was extracted and digested with a methylation-sensitive (HpaII) or methylation-insensitive $(M s p I)$ restriction enzyme, and the promoter regions of the Fas, MICA and MICB genes were amplified by PCR. The band in lane 6 indicates the amplified product of the promoter region of the MICA or MICB gene without treatment with either of the restriction enzymes.
VPA inhibited the hydralazine-induced increase in the production of soluble Fas although VPA with hydralazine cooperated to increase the cell-surface Fas. Therefore, the effect of VPA on the production of soluble Fas may be ascribed to its effect on the alternative splicing of the Fas gene transcript, although the mechanism(s) remain unknown.

Hydralazine showed no significant effect on the production of soluble MICA and MICB, while VPA decreased their production. Soluble MICA and B are produced by the proteolytic cleavage of cell-surface MICA and MICB by proteases, including matrix metalloproteinases (MMPs) $(9,10)$, and human osteosarcomas have been reported to produce MMP-2 and $-9(33,34)$. Since it has been reported that HDAC inhibitors including VPA down-regulate the activity of MMPs in gastric, thyroid and lung cancer cells (36-38), the inhibitory effect of VPA on the production of soluble MICs may be ascribed to its inhibitory action on the activity of MMPs. This possibility is now under investigation.

VPA inhibited the growth of osteosarcoma cells, but hydralazine had no significant effect on their growth. Although hydralazine was reported to inhibit the growth of human 
cervical cancer cells (39), Chavez-Blanco et al (40) reported that the growth of 7 human tumor cell lines of different origins were inhibited by VPA $(1.0 \mathrm{mM})$ but not by hydralazine $(10 \mu \mathrm{M})$, and that hydralazine, in combination with VPA, produced further inhibitory effects in only 2 of the 7 cell lines. Therefore, it is likely that the inhibitory effect of hydralazine on the growth of cells varies depending on tumor types.

The present study showed that VPA and hydralazine cooperated to increase the expression of Fas and MICA and B on the surface of human osteosarcoma cells, and sensitized them to Fas-mediated and NK cell-mediated death. They also decreased the production of soluble MICA and B by osteosarcoma cells. Therefore, a combination of VPA and hydralazine may be valuable for augmenting the efficiency of immunotherapy for osteosarcomas.

\section{Acknowledgements}

This study was in part supported by a Grant-in-Aid for Young Scientists (B) (23792159) from the Ministry of Education, Culture, Sports, Science, and Technology of Japan, Strategic Program Grant for Research Infrastructure Development in Private Institutes, and Grant-in-Aid for Promotion of Technical Seeds in Advanced Medicine, Hyogo College of Medicine.

\section{References}

1. Waldhauer I and Steinle A: NK cells and cancer immunosurveillance. Oncogene 27: 5932-5943, 2008.

2. Nausch $\mathrm{N}$ and Cerwenka A: NKG2D ligands in tumor immunity. Oncogene 27: 5944-5958, 2008.

3. Kägi D, Vignaux F, Ledermann B, Bürki K, Depraetere V, Nagata S, Hengartner H and Golstein P: Fas and perforin pathways as major mechanisms of T cell-mediated cytotoxicity. Science 265: 528-530, 1994.

4. Arase H, Arase N and Saito T: Fas-mediated cytotoxicity by freshly isolated natural killer cells. J Exp Med 181: 1235-1238, 1995.

5. Nagata S: Apoptosis by death factor. Cell 88: 355-365, 1997.

6. Cheng J, Zhou T, Liu C, Shapiro JP, Brauer MJ, Kiefer MC, Barr PJ and Mountz JD: Protection from Fas-mediated apoptosis by a soluble form of the Fas molecule. Science 263: 1759-1762, 1994.

7. Cascino I, Fiucci G, Papoff G and Ruberti G: Three functional soluble forms of the human apoptosis-inducing Fas molecule are produced by alternative splicing. J Immunol 154: 2706-2713, 1995.

8. Owen-Schaub LB, Angelo LS, Radinsky R, Ware CF, Gesner TG and Bartos DP: Soluble Fas/APO-1 in tumor cells: a potential regulator of apoptosis? Cancer Lett 94: 1-8, 1995.

9. Salih HR, Rammensee HG and Steinle A: Cutting edge: downregulation of MICA on human tumors by proteolytic shedding. J Immunol 169: 4098-4102, 2002

10. Salih HR, Goehlsdorf D and Steinle A: Release of MICB molecules by tumor cells: mechanism and soluble MICB in sera of cancer patients. Hum Immunol 67: 188-195, 2006

11. Boutet P, Agüera-González S, Atkinson S, Pennington CJ, Edwards DR, Murphy G, Reyburn HT and Valés-Gómets M: Cutting edge: the metalloproteinase ADAM17/TNF-alphaconverting enzyme regulates proteolytic shedding of the MHC class I-related chain B protein. J Immunol 182: 49-53, 2009.

12. Groh V, Wu J, Yee C and Spies T: Tumour-derived soluble MIC ligands impair expression of NKG2D and T-cell activation. Nature 419: 734-738, 2002.

13. Raffaghello L, Prigione I, Airoldi I, Camoriano M, Levreri I, Gambini C, Pende D, Steinle A, Ferrone S and Pistoia V: Downregulation and/or release of $\mathrm{NKG} 2 \mathrm{D}$ ligands as immune evasion strategy of human neuroblastoma. Neoplasia 6: 558-568, 2004.

14. Märten A, Von Lilienfeld M, Büchler MW and Schmidt J: Soluble MIC is elevated in the serum of patients with pancreatic carcinoma diminishing gammadelta $\mathrm{T}$ cell cytotoxicity. Int J Cancer 119: 2359-2365, 2006.
15. Yoo CB and Jones PA: Epigenetic therapy of cancer: past, present and future. Nat Rev Drug Discov 5: 37-50, 2006.

16. Kristensen LS, Nielsen HM and Hansen LL: Epigenetics and cancer treatment. Eur J Pharmacol 625: 131-142, 2009.

17. Armeanu S, Bitzer M, Lauer UM, Venturelli S, Pathil A, Krusch M, Kaiser S, Jobst J, Smirnow I, Wagner A, Steinle A and Salih HR: Natural killer cell-mediated lysis of hepatoma cells via specific induction of NKG2D ligands by the histone deacetylase inhibitor sodium valproate. Cancer Res 65: 6321-6329, 2005.

18. Schmudde M, Braun A, Pende D, Sonnenmann J, Kiler U, Beck JF, Moretta L and Bröker BM: Histone deacetylase inhibitors sensitize tumour cells for cytotoxic effects of natural killer cells. Cancer Lett 272: 110-121, 2008.

19. Zhang C, Wang Y, Zhou Z, Zhang J and Tian Z: Sodium butyrate upregulates expression of NKG2D ligand MICA/B in HeLa and HepG2 cell lines and increases their susceptibility to NK lysis. Cancer Immunol Immunother 58: 1275-1285, 2009.

20. Poggi A, Catellani S, Garuti A, Pierri I, Gobbi M and Zocchi MR: Effective in vivo induction of NKG2D ligands in acute myeloid leukaemias by all-trans-retinoic acid or sodium valproate. Leukemia 23: 641-648, 2009.

21. Yamanegi K, Yamane J, Kobayashi K, Kato-Kogoe N, Ohyama H, Nakasho K, Yamada N, Hata M, Nishioka T, Fukunaga S, Futani $\mathrm{H}$, Okamura $\mathrm{H}$ and Terada N: Sodium valproate, a histone deacetylase inhibitor, augments the expression of cell-surface NKG2D ligand, MICA/B, without increasing their soluble forms to enhance susceptibility of human osteosarcoma cells to NK cell-mediated cytotoxicity. Oncol Rep 24: 1621-1627, 2010.

22. Insinga A, Monestiroli S, Ronzoni S, Gelmetti V, Marchesi F, Viale A, Altucci L, Nervi C, Minucci S snd Pelicci PG: Inhibitors of histone deacetylases induce tumor-selective apoptosis through activation of the death receptor pathway. Nat Med 11: 71-76, 2005.

23. Angelucci A, Valentini A, Millimaggi D, Gravina GL, Miano R, Dolo V, Vicentini C, Bologna M, Federici G and Bernardini S: Valproic acid induces apoptosis in prostate carcinoma cell lines by activation of multiple death pathways. Anticancer Drugs 17: 1141-1150, 2006.

24. Imai T, Adachi S, Nishijo K, Ohgushi M, Okada M, Yasumi T, Watanabe K, Nishikomori R, Nakayama T, Yonehara S, Toguchida J and Nakahata T: FR901228 induces tumor regression associated with induction of Fas ligand and activation of Fas signaling in human osteosarcoma cells. Oncogene 22: 9231-9242, 2003.

25. Watanabe K, Okamoto K and Yonehara S: Sensitization of osteosarcoma cells to death receptor-mediated apoptosis by HDAC inhibitors through downregulation of cellular FLIP. Cell Death Differ 12: 10-18, 2005.

26. Schuchmann M, Schulze-Bergkamen H, Fleischer B, Schattenberg JM, Siebler J, Weinmann A, Teufel A, Wörns M, Fischer T, Strand S, Lohse AW and Galle PR: Histone deacetylase inhibition by valproic acid down-regulates c-FLIP/CASH and sensitizes hepatoma cells towards CD95- and TRAIL receptormediated apoptosis and chemotherapy. Oncol Rep 15: 227-230, 2006.

27. Yamanegi K, Yamane J, Hata M, Ohyama H, Yamada N, KatoKogoe N, Futani H, Nakasho K, Okamura H and Terada N: Sodium valproate, a histone deacetylase inhibitor, decreases the secretion of soluble Fas by human osteosarcoma cells and increases their sensitivity to Fas-mediated cell death. J Cancer Res Clin Oncol 135: 879-889, 2009.

28. Petak I, Danam RP, Tillman DM, Vernes R, Howell SR, Berczi L, Kopper L, Brent TP and Houghton JA: Hypermethylation of the gene promoter and enhancer region can regulate Fas expression and sensitivity in colon carcinoma. Cell Death Differ 10: 211-217, 2003.

29. Tang KF, He CX, Zeng GL, Wu J, Song GB, Shi YS, Zhang WG, Huang AL, Steinle A and Ren H: Induction of MHC class I-related chain B (MICB) by 5-aza-2'-deoxycytidine. Biochem Biophys Res Commun 370: 578-583, 2008.

30. Johannessen CU and Johannessen SI: Valproate: past, present, and future. CNS Drug Rev 9: 199-216, 2003.

31. Spina E and Perugi G: Antiepileptic drugs: indications other than epilepsy. Epileptic Disord 6: 57-75, 2004.

32. Kandler MR, Mah GT, Tejani AM and Stabler SN: Hydralazine for essential hypertension. Cochrane Database Syst Rev 8: CD004934, 2010.

33. Cho HJ, Lee TS, Park JB, Park KK, Choe JY, Sin DI, Park YY, Moon YS, Lee KG, Yeo JH, Han SM, Cho YS, Choi MR, Park NG, Lee YS and Chang YC: Disulfiran suppresses invasive ability of osteosarcoma cells via the inhibition of MMP-2 and MMP-9 expression. J Biochem Mol Biol 40: 1069-1076, 2007. 
34. Xin ZF, Kim YK and Jung ST: Risedronate inhibits human osteosarcoma cell invasion. J Exp Clin Cancer Res 28: 105, 2009.

35. Venkataraman GM, Suciu D, Groh V, Boss JM and Spies T: Promoter region architecture and transcriptional regulation of the genes for the MHC class I-related chain A and B ligands of NKG2D. J Immunol 178: 961-969, 2007.

36. Lee KH, Choi EY, Kim MK, Kim KO, Jang BI, Kim SW, Kim SW, Song SK and Kim JR: Inhibition of histone deacetylase activity down-regulates urokinase plasminogen activator and matrix metalloproteinase-9 expression in gastric cancer. Mol Cell Biochem 343: 163-171, 2010.

37. Mitmarker EJ, Griff NJ, Grogan RH, Sarkar R, Kebebew E, Duh QY, Clark OH and Shen WT: Modulation of matrix metalloproteinase activity in human thyroid cancer cell lines using demethylating agents and histone deacetylase inhibitors. Surgery 149: 504-511, 2011.
38. Vinodhkumar R, Song YS, Ravikumar V, Ramakrishnan G and Devaki T: Depsipeptide a histone deacetylase inhibitor down regulates levels of matrix metalloproteinase 2 and 9 mRNA and protein expression in lung cancer cells (A549). Chem Biol Interact 165: 220-229, 2007

39. Song $Y$ and Zhang C: Hydralazine inhibits human cervical cancer cell growth in vitro in association with APC demethylation and re-expression. Cancer Chemother Pharmacol 63: 605-613, 2009.

40. Chavez-Blanco A, Perez-Plasencia C, Perez-Cardenas E, Carrasco-Legleu C, Rangel-Lopez E, Segura-Pacheco B, TajaChayeb L, Trejo-Becerril C, Gonzalez-Fierro A, Candelaria M, Cabrera G and Duenas-Gonzalez A: Antineoplastic effects of the DNA methylation inhibitor hydralazine and the histone deacetylase inhibitor valproic acid in cancer cell lines. Cancer Cell Int 6: 2, 2006. 